\title{
A chemistry set for life
}

\author{
Small molecules that selectively disrupt the proteins encoded by individual \\ genes could become powerful tools in functional genomics. Trisha Gura \\ explores the nascent but highly promising field of chemical genetics.
}

$\mathrm{N}$ ow would seem to be a good time to embark on a career in genetics. Demand will be high as laboratories the world over get to grips with the huge task of assigning functions to the thousands of genes in the human genome - and those of a growing number of model organisms. But the era of functional genomics could also create a rash of employment opportunities in chemistry.

Geneticists are realizing that chemists can make an important contribution by designing specific small molecules that can selectively disable particular genes and their protein products. Indeed, by combining genetics and chemistry, several research teams have managed to devise specific and reversible 'switches' to investigate the role of genes that have, until now, defied functional analysis.

This week's Nature includes a particularly elegant demonstration of this 'chemical genetic' approach ${ }^{1}$. By introducing mutations into genes that encode for members of a class of enzymes known as kinases, a team led by chemist Kevan Shokat of the University of California at San Francisco can switch off individual kinases using specially designed chemical inhibitors. This is an important advance: kinase enzymes are involved in countless cell-signalling pathways, but biologists have lacked the tools to disable individual members of the family and so discern their precise functions. "Everybody who studies kinases will start using this," predicts Doug Kellogg, a cell biologist at the University of California, Santa Cruz, who is now collaborating with Shokat's team.

With other groups making good progress in applying similar approaches to different genes, chemical genetics should soon be yielding a rich harvest. "Our goal is to dissect the function of organisms and cells by having a small molecule partner for every gene product," says Stuart Schreiber of the Institute of Chemistry and Cell Biology (ICCB) at Harvard University. In addition to advancing our knowledge of fundamental biology, this approach could spur drug development — in many cases, chemicals that inhibit par- ticular proteins could be developed into candidate drugs.

Not surprisingly, the US National Institutes of Health is starting to take an interest. Over the past two years, its largest centre, the National Cancer Institute (NCI) in Bethesda, Maryland, has awarded more than \$6 million to the ICCB and five other institutes working at the interface between chemistry and biology. "The discovery, modification and annotation of small molecules in terms of their ability to probe and perturb biological targets will be one of the central tools for the post-genomic era," says Richard Klausner, director of the NCI.

\section{Family values}

Shokat and his colleagues were drawn to chemical genetics through sheer necessity. Kinases participate in cell-signalling pathways by adding phosphate groups to other molecules. For their activity, they require adenosine triphosphate (ATP), a biochemical that acts as the energy 'currency' of the cell. But individual kinases guard the secrets of their function closely. If one kinase gene is mutated or deleted, disabling its enzyme product, other kinases often compensate over the course of the organism's development to assume the function of the disabled

Budding out: chemical genetics has shed light on an enzyme crucial to yeast reproduction. enzyme. And although several chemicals can inhibit kinases, they are not specific to individual members of the enzyme class you can disable the kinase you are interested in, but you will also knock out a handful of other kinases at the same time.

What Shokat needed was a way to disable individual kinases without giving cells enough time to call up a stand-in. The researchers began by mutating a selected kinase gene to change the site on the enzyme that binds to ATP. This enlarged the ATPbinding pocket but otherwise did not affect the enzyme's normal function. Then came the chemistry. The researchers added a variety of bulky chemical groups to small molecules already known to inhibit kinases, searching for a molecule that could slot neatly into the enlarged site and stop ATP from activating the enzyme.

Through this marriage of chemistry and genetics, Shokat and

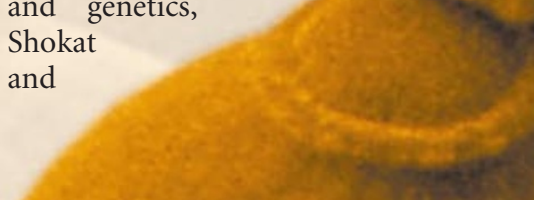


his colleagues turned a non-specific kinase inhibitor into a highly specific one. "We combined genetics and chemistry in a new way that allows you to study kinases with a very regulatable switch," says Shokat. And the switch is reversible, unlike a permanent mutation. Once the inhibitor is removed from the fluid bathing the cells, it is gradually replaced by ATP in the affected kinase, which turns the enzyme back on.

The team reported their first successes in 1998, disabling two kinases ${ }^{2}$. They have since expanded on this work ${ }^{3,4}$, and their Nature paper provides evidence that the approach can be applied to any kinase ${ }^{1}$. Using chemical derivatives of two different kinase inhibitors, Shokat's team has, to date, disabled kinases from five different sub-families, working with the yeast Saccharomyces cerevisiae, mammalian cells and mouse embryos.

Already, Shokat and his colleagues are uncovering information on the function of kinases. With Dave Morgan, a cell biologist at the University of California at San Francisco, they have gained fresh insights into the function of Cdc28, the major kinase regulating the cell cycle in yeast. And in the latest issue of Nature Cell Biology $y^{5}$, working in collaboration with David Drubin of the University of California at Berkeley, they identify the role of a kinase called Cla4p in yeast. The kinase is needed to start budding, a form of cell division in which a daughter cell grows out from its parent, before eventually pinching off and going its separate way.

\section{Slot machinery}

Other groups, including Peter Schultz and his colleagues at the Scripps Research Institute in La Jolla, California, are using similar chemical strategies to investigate interactions between proteins. The Scripps team has mutated the genes for human growth hormone and its receptor so that the two proteins produced can no longer bind to one another. To do this, they engineered mutations that replaced the amino acids tryptophan and threonine with glycine. These amino acids all have similar backbones, but tryptophan has an additional side-group called an indole. Replacing tryptophan with glycine creates a cavity between hormone and receptor. The researchers reasoned that chemically synthesized indole derivatives would fill this cavity and restore the interaction between hormone and receptor. The strategy worked: Schultz and his colleagues screened a relatively small library of some 200 different compounds and found one small molecule that did the job perfectly ${ }^{6}$. Schultz believes this approach could be used to study the function of a wide range of different protein-protein and protein-nucleic acid interactions.

Meanwhile, Thomas Kodadek of the University of Texas Southwestern Medical

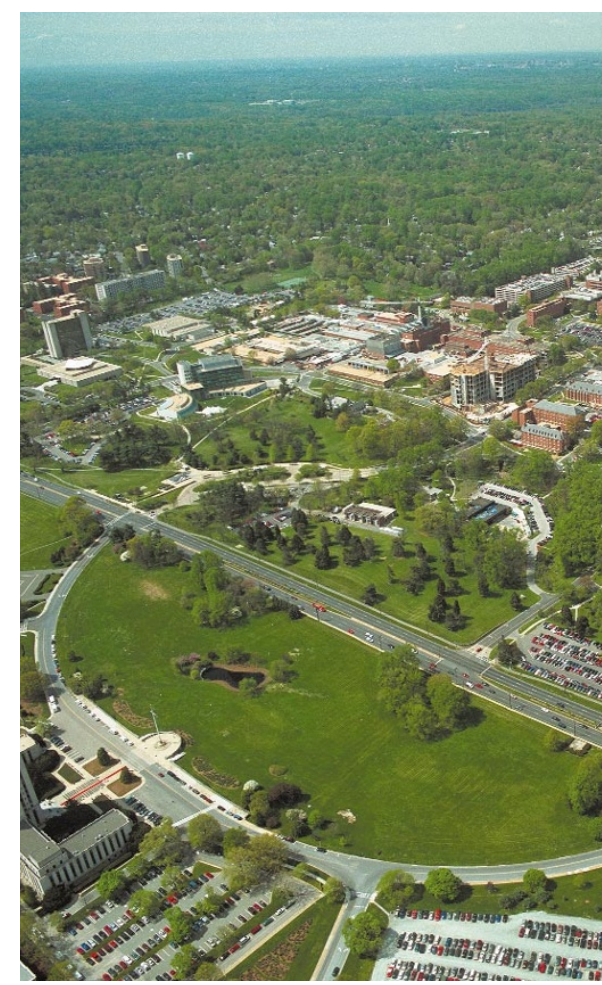

Powerhouse: the National Institutes of Health (above) is backing chemical genetics as a central tool, with Klausner as its champion.

Center at Dallas is looking for small molecules that mimic specific transcription factors. These proteins switch genes on or off by binding to their regulatory sequences. But before transcription factors can do their work, DNA has to be unwound from proteins called histones found inside the cell's chromosomes. So, in addition to transcription factor mimics, Kodadek is looking for small molecules that can trigger this unwinding. The two molecules could then be tethered together. With his colleague Carilee Denison, Kodadek outlined this strategy in a 1998 paper $^{7}$, arguing that it should allow the expression of a "significant fraction" of the genes in the human genome to be controlled artificially. "We are trying to develop a platform technology that solves the problem of how to turn genes on," says Kodadek.

\section{Forward thinking}

All of these approaches are similar in concept to established techniques of 'reverse genetics', in which particular proteins are targeted by altering the genes that encode them. But equally valuable are 'forward genetic' approaches, in which researchers mutate the genome at random, screen for mutants with biologically interesting defects, and then work out which gene is involved. This strategy is also being mimicked by chemical genetics. "We're trying to emulate all the principles of genetics using small molecules," says Schreiber.

This effort marks a return to old-fashioned methods of drug discovery. More than

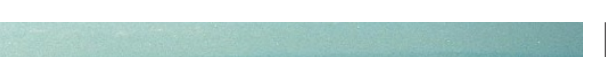


drug discovery process," says NCI director Klausner. But the main focus in many labs is more fundamental biology: the researchers are looking for chemicals with specific effects that might reveal the function of individual genes.

Recent advances in chemical synthesis have facilitated chemical genetics by making it much easier to produce a huge selection of small molecules. Combinatorial chemistry, which builds libraries of compounds by combining smaller molecular building blocks in different configurations, has been used since the early 1990s to generate biologically active molecules ${ }^{8}$. Also, chemists are now developing algorithms that allow them to plan specific synthetic pathways needed to produce a diversity of structurally complex small molecules ${ }^{9}$.

Some labs are also sampling the wares of cash-strapped chemistry institutes from the former Soviet Union, some of which are surviving by selling their huge libraries of compounds to researchers in the West through a company called AsInEx, based in Moscow. "Now it is possible for a biologist to hook up with a chemist's tens or hundreds of thousands of molecules," says Craig Crews, a cell biologist at Yale University in New Haven, Connecticut.

Technologies emerging from genomics are also boosting the field. Robots developed to handle the huge throughput of biological samples needed for genome sequencing can
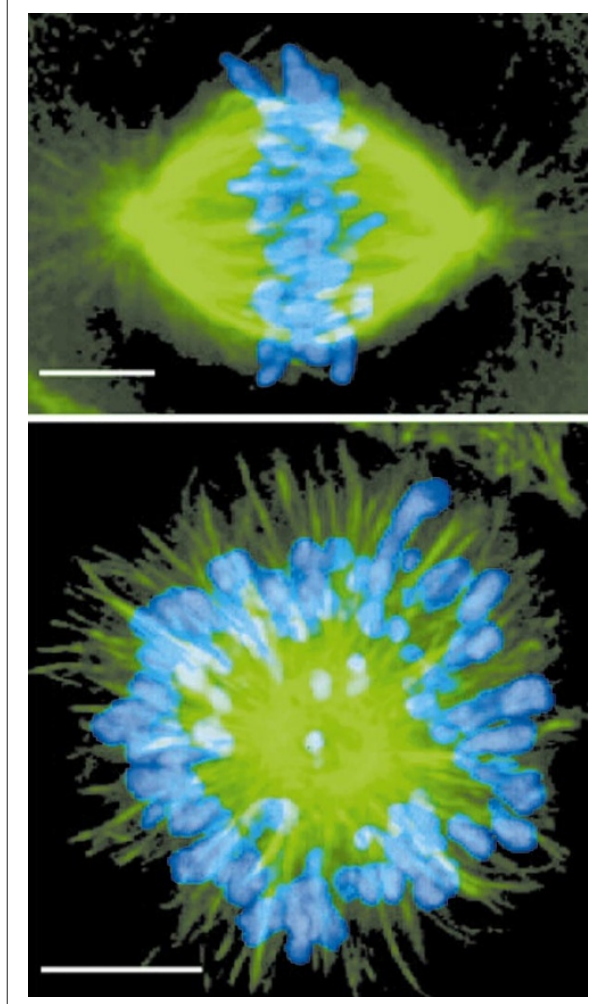

Star performer: in normal cell division (top), the chromosomes (blue) move along microtubules (green) in an orderly fashion. But adding monastrol disrupts the system (bottom).

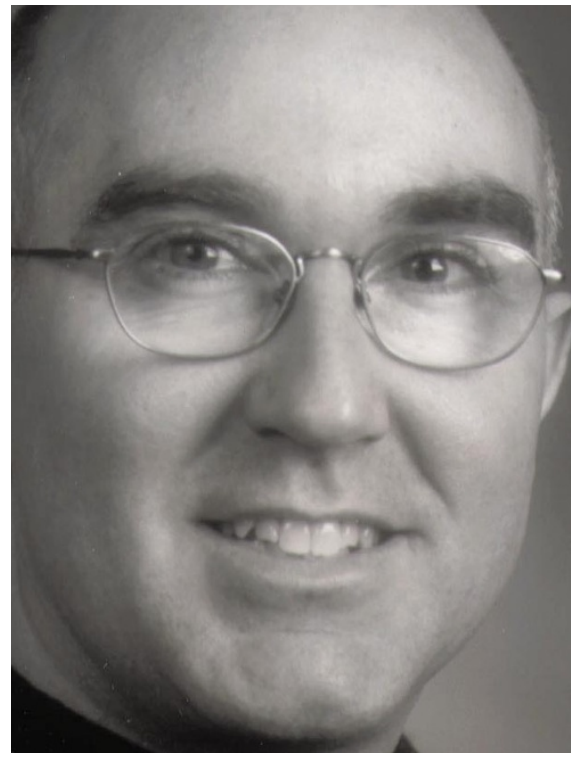

Schreiber: mimics genetics with small molecules.

be adapted to screen for the effects on cells of thousands of different chemicals. Microarrays, in which libraries of nucleic acids or proteins are immobilized on glass slides, are among the most important tools in functional genomic analysis - and this idea is also being adapted to chemical genetics. Schreiber and his colleagues, for instance, have created microarrays 'printed' with libraries of small molecules ${ }^{10}$. Extracts of cells can be added to these chips as a 'prescreen' in chemical-genetic projects. This allows the researchers to home in on compounds that bind to cellular proteins, reducing the burden of running time-consuming biological assays.

\section{Selection process}

Perhaps the most striking demonstration of the power of forward chemical genetics has come from a collaboration between Timothy Mitchison's lab at Harvard Medical School and Schreiber's group at the ICCB. "The whole idea was to find membrane-permeable compounds that affect the proteins required in cell division," explains Thomas Mayer, the postdoc who led the project ${ }^{11}$. "Then we can use these compounds to the study the function of the proteins."

Mayer and his colleagues began with a library of 16,320 small molecules. If cells are arrested in the early phases of division, they build up high levels of a phosphorylated form of a protein called nucleolin, which can be detected using a light-emitting antibody that binds to it. Using this screen, the Harvard researchers identified 139 small molecules that inhibited cell division. Fifty-three of these targeted a protein called tubulin that forms microtubules - the 'tracks' along which replicated chromosomes move to either end of a cell as it prepares to divide.

Mayer wanted to disable proteins whose role in cell division was unknown, so he tested the remainder of the small molecules by adding them to mammalian kidney cells and watching the effects on cell division with a microscope after staining them with fluorescently tagged antibodies that bound to both microtubules and chromosomes. One compound, which the researchers dubbed 'monastrol', caused the microtubules to form into a central star-like structure, rather than the usual spindle running from end to end of the cell.

The team went on to discover that monastrol's target is a protein called Eg5. This protein is known to be involved in spindle formation, but researchers do not understand its precise function. With inhibitor and target in hand, Mayer and his colleagues are now adding monastrol to frog cell extracts to dissect the dynamics of spindle formation and the control of cell division ${ }^{12}$.

In future, chemical genetics could be applied to developmental studies, for instance in model organisms such as the zebrafish. The advantage of the chemical approach is that it allows genes to be switched off later in an organism's development, says Schreiber. Conventional approaches often induce genetic mutations that are fatal in early development, making it impossible to see any secondary functions a gene might have, which would appear only later on.

If anything is going to hold chemical genetics back, it is most likely to be the cultural difficulty of getting biologists and chemists to collaborate. "It's a tribal clash," says Kodadek. "Some biologists want to hire a bunch of synthetic organic chemists just to make whatever compounds they tell them to." Not surprisingly, the most talented chemists want to work on their own research projects, rather than being at a biologist's beck and call.

Now, as efforts proceed to merge the two disciplines, students are being trained in both fields. "One of the things we are going to observe in the next few years is a broadening of the base of researchers who participate in chemistry,"Klausner predicts. If he is correct, these multi-skilled individuals could hold some of the keys to deciphering the human genome, and help to discover tomorrow's life-saving drugs.

Trisha Gura is a freelance writer in Cleveland, Ohio.

1. Bishop, A. C. et al. Nature 407, 395-401 (2000).

2. Bishop, A. C. et al. Curr. Biol. 8, R257-R266 (1998)

3. Liu, T., Shah, K., Yang, F., Witucki, L. \& Shokat, K. M. Chem. Biol. 5, 91-101 (1998).

4. Bishop, A. C. et al. J. Am. Chem. Soc. 121, 627-631 (1999).

5. Weiss, E. L., Bishop, A. C., Shokat, K. M. \& Drubin, D. G. Nature Cell Biol. 2, 677-685 (2000).

6. Guo, Z., Zhou, D. \& Schultz, P. G. Science 288, 2042-2045 (2000)

Denison, C. \& Kodadek, T. Chem. Biol. 5, R129-R145 (1998).

8. Bunin, B. A. \& Ellman, J. A. J. Am. Chem. Soc. 114, 10997-10998 (1992)

9. Schreiber, S. Science 287, 1964-1969 (2000).

10. MacBeath, G., Koehler, A. N. \& Schreiber, S. L. J. Am. Chem. Soc 121, 7967-7968 (1999).

11. Mayer, T. U. et al. Science 286, 971-974 (1999).

12. Kapoor, T. M., Mayer, T. U, Coughlin, M. L. \& Mitchison, T. J. J. Cell Biol. 150, 975-988 (2000). 